\title{
Errata: Choosing optimal wavelength for photodynamic therapy of port wine stains by mathematic simulation
}

Ying Wang, Ying Gu, Zhaohui Zuo, and Naiyan Huang

Chinese People's Liberation Army General Hospital, Department of Laser Medicine, Beijing 100853, China

[DOI: $10.1117 / 1.3650768]$

This article [J. Biomed. Opt. 16, 098001 (2011)] was originally published online on 1 September 2011 an error in Figure 7, where the labels pointing to the HMME and hemoglobin lines were reversed. The corrected figure appears below.

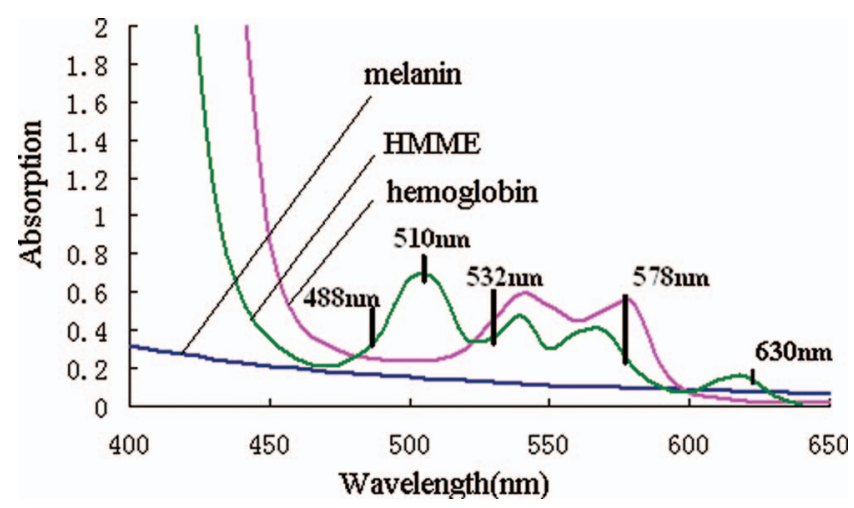

Fig. 7

All versions of the article were corrected on 27 September 2011. The article appears correctly in print. 